Bangladesh J. Plant Taxon. 13(1): 29-40, 2006 (June)

\title{
PLEUROCARPOUS MOSSES OF BANGLADESH : SYMPHYODONTACEAE AND AMBLYSTEGIACEAE
}

\author{
Hamida Khatun and Syed HadiuzZaman \\ Department of Botany, University of Dhaka, Dhaka-1000, Bangladesh
}

Key words : Pleurocarpous moss, Hookeriales, Hypnobryales, Symphyodontaceae

Amblystegiaceae, Bangladesh

\begin{abstract}
A taxonomic account of six species of Pleurocarpous mosses of Bangladesh of the families Symphyodontaceae and Amblystegiaceae under the order Hookeriales and Hypnobryales respectively are given .
\end{abstract}

\section{Introduction}

The family Symphyodontaceae is being represented in Bangladesh by only a single genus with two species, and the family Amblystegiaceae is represented by two genera and four species of the order Hookeriales. During detailed studies on the Pleurocarpous mosses of Bangladesh Khatun and Hadiuzzaman (1994, 1995, 2003, 2004, 2005) found that among the different groups of these mosses, the order Hookeriales is not so common. Two species of the genus Symphyodon of the family Symphyodontaceae are being described in this present paper, namely S. orientales and S. erraticus. Gangulee (1977) described eight species of the genus Symphyodon from eastern India and adjacent regions, but he did not give any information on the occurrence of this genus in Bangladesh. He also mentioed S. orientalis is endemic to Upper Assam and S. erraticus is endemic to Indo-Cylon but both species were found in Bangladesh.

Chaetomitrium philippenense Mont. of the family Hookeriaceae under Hookeriales was reported earlier from Bangladesh by Tixier (1967) from Kaptai, Rangamati on twigs in a checklist only, but none from the genus Symphyodon. Very recently Banu- Fattah (2005) reported Distichophyllum schmidtii Broth. of the family Hookeriaceae. This moss is also rare and earlier it was only collected by Sinclair (1955) from Kalatuli, Cox's Bazar.

A previous study of Khatun and Hadiuzzaman (2005) revealed that two families of Pleurocarpous mosses of the order Hypnobryales, namely, Thuidiaceae and Brachytheciaceae are present in Bangladesh. Another family Amblystegiaceae is being described in the present paper. This family is represented by two genera, namely, Cratoneuron and Campylium. The genus Cratoneuron is represented by a single species, namely, $C$. filicinum. The genus Campylium is represented by three species, namely $C$. gollanii, C. sommerfeltii and C. stellatum. Tixier (1967) reported several Pleurocarpous mosses from Bangladesh, but not one from the family Amblystegiaceae. Gangulee (1978) reported two species of the genus Cratoneuron and two species of the genus 
Campylium of the family Amblystegiaceae from the eastern India and adjacent regions. Chopra (1975) also reported four species of Cratoneuron and four species of Campylium. These authors did not mention the occurrence of these taxa from Bangladesh territory. In the present paper two species of Symphyodon of the family Symphyodontaceae of the order Hookeriales and one species of Cratoneuron, three species of Campylium, of the same order are being fully described and illustrated along with the key to the species. Their distribution in Bangladesh has also been recorded.

Order: Hookeriales; Family: Symphyodontaceae

Genus: Symphyodon Mont in Ann. Sc. Nat. Bot. ser. 2, 16 : 279 (1941)

Key to the species of Symphyodon

1. Leaves ovate, a spinose row of shorter and broader cells at top margin

S. orientalis

- $\quad$ Leaves ovate-lanceolate, no spinose row of shorter and broader cells at top

marginal cells

S. erraticus

1. Symphyodon orientalis (Mitt.) Broth. ex Par. in coll. 33 (1909)

(Fig. 1)

Stereodon orientalis Mitt. in Musci Ind. Or. : 111(1859)

Plant slender, yellow green, main stem creeping, branches irregularly pinnate, top of the branches fasciculate, became yellowish with age, branches more or less patent, branches and leaves curled when dry. 2 to $3 \mathrm{~cm}$ or more long. Leaves more or less complanate and more or less distichous, leaves dense, erectopatent, ovate, slightly concave, gradually narrowed towards tip, acute, sometimes slightly falcate, more or less 1 to $1.5 \mathrm{~mm}$ long, 0.4 to $0.5 \mathrm{~mm}$ wide, margin entire but apical portion dentate, costa short, double, more or less equal, sometimes unequal. Leaf cells linear to linear-elongate, tip cells irregularly linear up to c. $80 \times 5 \mu \mathrm{m}$, border cells of tip more broader and shorter, middle cells elongated linear, c. $86 \times 4.5 \mu \mathrm{m}$, alar not distinctly differentiated but cells irregularly rectangular or irregularly quadrate at basal attachment, c. $20 \times 8.5 \mu \mathrm{m}$, all cells show one papillose development at its tip, cell wall irregularly thickened but more or less thin. Main stem leaves and branch stem leaves more or less similar. Sporophyte on main shoot, sometimes on branch shoot. Perichaetial leaves elongated, narrow c. $2.5 \mathrm{~mm}$ long. Seta erect c. $2 \mathrm{~cm}$ long, upper portion rough, Capsule curved from apophysis, elongated-cylindric, horizontal, c. $1 \mathrm{~mm}$ long, and $0.5 \mathrm{~mm}$ in diameter, exothecial cells irregularly quadrate containing stomata. Peristome teeth normal, double, exostome yellow-brown, transverse striolation present, c. $3 \mu \mathrm{m}$ high, endostome is same height and golden-yellow in colour.

Specimens examined: Maulvi Bazar: Srimangal, Lawacherra Forest, on soil, Saiful Islam, 03.03.92,325; Sirajganj: Tarash, on soil, Ferdous Alam, 06.12.98, 1443; Sylhet: Golapganj, on soil,Hamida Khatun, 06.01.96, 367 


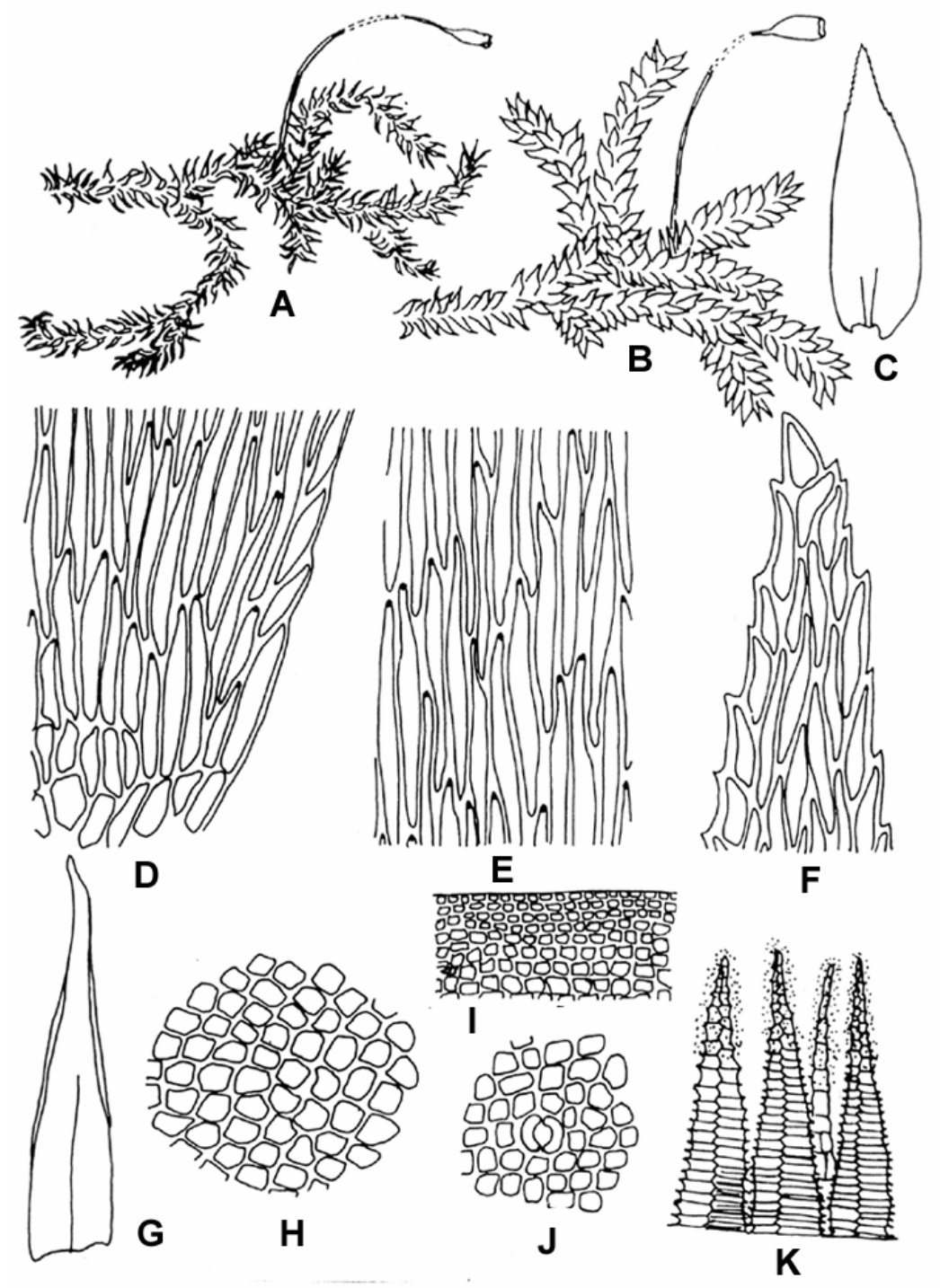

Fig. 1. Symphyodon orientalis (Mitt.) Broth. A. dry plant $(\times 6.67)$, B. wet plant $(\times 6.67)$, C. leaf $(\times 24)$, D. basal laminal cells $(\times 300)$, E. middle laminal cell $(\times 300)$, F. apical laminal cells $(\times 300)$, G. perichaetial leaf $(\times 24)$, H. exothecial cells of the capsule $(\times 133)$, I. mouth cells of the capsule $(\times 133)$, J. exothecial cells showing stomata $(\times 133)$, K. peristome teeth $(\times 133)$.

2. Symphyodon erraticus (Mitt.) Jaeg. in Ber. S.Gall. Naturw. Ges. 1876-77: 296 (1878)

(Fig. 2)

Stereodon erraticus Mitt. in Musci. Ind. Or . :111 (1859)

Reddish to blackish yellow, robust plants. Main stem creeping, secondary shoots spreading, pinnately branched, branches some times bi- or tri-pinnate, $10 \mathrm{~cm}$ or more long. Leaves erectopatent to spreading, ovate lingulate, slightly concave, sometimes one 
side of the leaf revolute, c. $1.5 \mathrm{~mm}$ long and $0.5 \mathrm{~mm}$ broad, apex acute, margin dentate from middle to top, sometimes also recurved below, somewhat asymmetric. Costa double, short, unequal. Leaf cells very linear elongate c. $55 \times 5 \mu \mathrm{m}$ at middle, tip cells little shorter than middle cells and c. $35.9 \times 5 \mu \mathrm{m}$, mostly showing a paiplla at upper angle, cells at attachment broader, laxer, quadrate below, and c. $20.5 \times 20 \mu \mathrm{m}$. Main stem leaves and branch leaves more or less similar but branch leaves little smaller, more concave, more ovate than main stem leaves. Sporophyte not found.

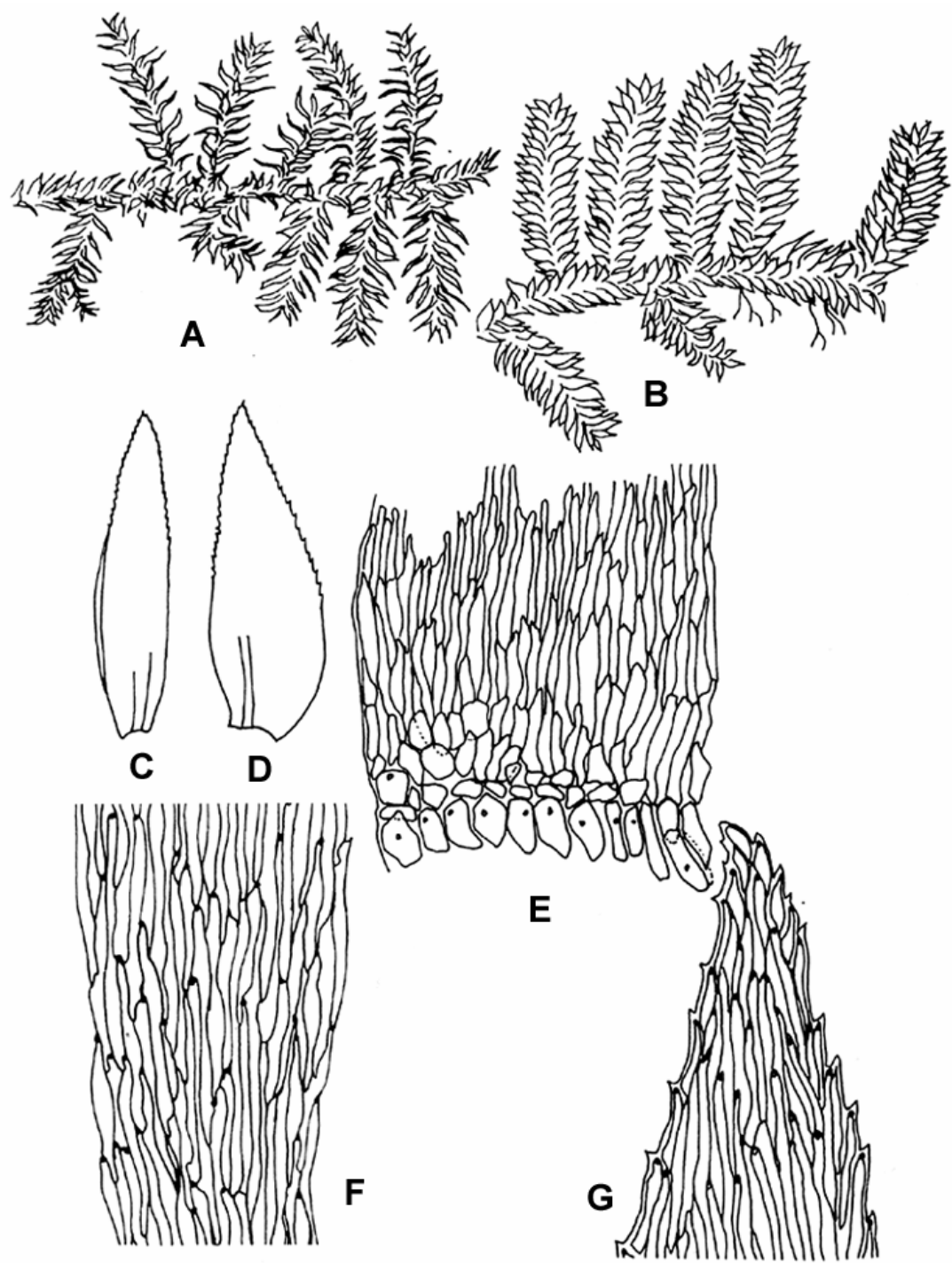

Fig. 2. Symphyodon erraticus (Mitt.) Jaeg. A. dry plant $(\times 6.67)$, B. wet plant $(\times 6.67)$, C, D. leaves $(\times 24)$, E. basal laminal cells $(\times 180)$, F. middle laminal cell $(\times 180)$, G. apical laminal cells $(\times 180)$. 
Specimens examined: Chittagong: Chittagong University, on the bark of tree, Kishore Kumar, 11.08.94, 341; Maulvi Bazar: Lawacherra Forest, Sandy soil, Hamida Khatun, 23.02.92, 325; Sylhet : Airport Road, on soil, Hamida Khatun, 06.01.96, 367

Order: Hypnobryales; Family: Amblystegiaceae

Genus: Cratoneuron (Sull.) Spruce. in Cat. Musc. Amaz. And.: 21 (1867)

3. Cratoneuron filicinum (L. ex Hedw.) Spruce. Cat. Musc. Amaz . And ., P. 21, 1867

Hypnum filicinum L. ex Hedw., in Sp. Musc., P 285, 1801.

(Fig. 3)

H.compressum Brid. in Musc. Rec., 2(2): 58 (1801).

Robust, medium-sized green to golden-green plants in dense tufts. Main stem usually ascending (prostrate, some branches errect), densely tomentose, c. 6-11 cm. long. Stems spreading or more often, crowded, irregularly or more typically pinnately branched. Paraplyllia present, variable in shape. Stem leaves erect or erect spreading or sometimes secund or slightly falcato-secund, ovate-lanceolate to broadly ovate, rather abruptly acuminate, the acumen usually not flexuose or twisted, decurrent, margins often slightly recurve at extreme base, mildly serrulate nearly all around except extreme base. Costa single, ending below tip c. $1.42 \mathrm{~mm} \times 0.7 \mathrm{~mm}$. Leaf cells also reported to be variable. In this case cells smooth, firm-walled, shortly oblong, sometimes oblong-rhomboidal about $3: 6$ to $6: 1$ i. e, $(40 \times 10 \mu \mathrm{m})$, cells at next to the tip shortly oblong, at extreme tip slightly elongated rhomboidal up to c. $36 \times 11 \mu \mathrm{m}$, middle cells show variation, sometimes small or sometimes large elongated-rhomboid c. $26.4 \times 10.8 \mu \mathrm{m}$. Alar cells abruptly differentiated, hyaline or yellow-brown, irregularly rectangular more or less thick walled c. $28 \times 12 \mu \mathrm{m}$ in concave groups. Branch leaves shorter and narrower, crowded erectopatent (erect with flexuose or out-spread tips when dry), lanceolate acuminate, more or less with falcate tips, up to $1.25 \mathrm{~mm}$ long. Costa single, ending below tip likely to be percurrent, cells as stem leaf. Sporophyte not found.

Specimen examined : Pabna: Raghunathpur, on the bark of tree, Luna Ahmed, 03.03.94, 193.

Genus: Campylium (Sull.) Mitt. in J. Linn. Soc. Bot., 12 : 631 (1869)

\section{Key to the species of Campylium}

1. Leaves ovate-lanceolate, more or less acute

C. gollanii

- Leaves not so but, from an ovate base narrowed into a channelled acumen

2. Leaves erect spreading to spreading, leaf ovate, near the apex secund

C. sommerfeltii

- $\quad$ Leaves spreading from an suberect base , leaf cordate ovate, leaf tip not so

C. stellatum 


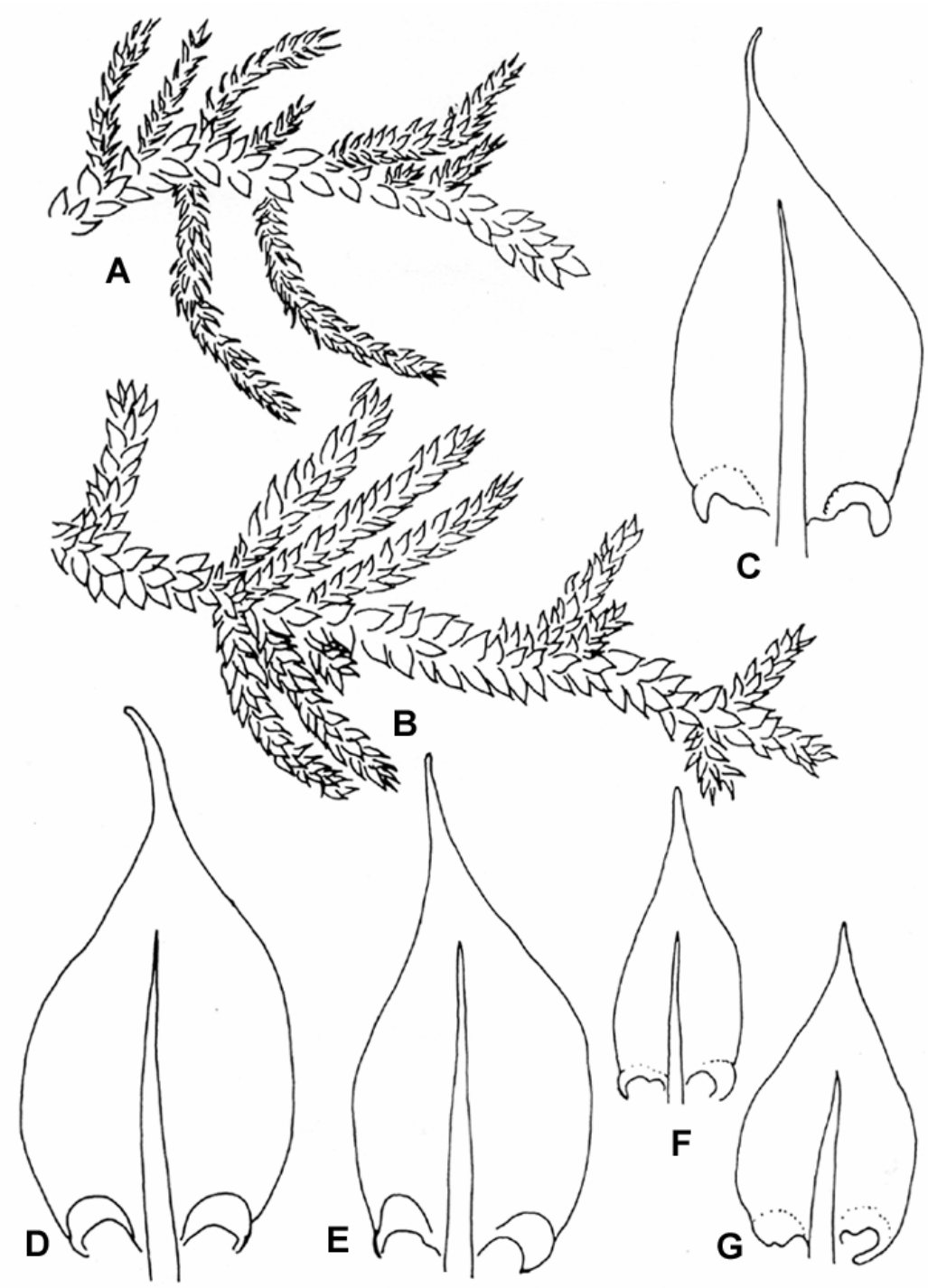

Fig. 3. Cratoneuron filicinum (Hedw.) Spruce. A. dry plant (×6.67), B. wet plant $(\times 6.67)$, C-G. larger and smaller leaves $(\times 24)$.

4. Campylium gollanii C. Muell. ex Vohra in Bull. Bot. Surv. India 12 (1-4) : 101. 1970.

(Fig. 4).

Dioecious, small plants, glossy, golden green, uaually corticolous and loosely tufted. Main stem creeping, branches ascending short arched, pinnate, dense, smooth up to 2.5 $\mathrm{cm}$ long and $0.5 \mathrm{~cm}$ wide with leaves. Leaves erect spreading to spreading, ovatelanceolate, margin slightly involute that makes the leaf little concave, apex narrowed into a long and fine acumen, margin entire, up to $1.25 \mathrm{~mm}$ long and $0.29 \mathrm{~mm}$ broad. Costa 
short, double, unequal, sometimes indistinct. Alar cells quadrate to sub-rectangular sometimes inflated, incrassate c. $22.99 \times 7.99 \mu \mathrm{m}$, smooth, not porose, middle cells narrowly linear, fusiform, c.78.7 $\times 3 \mu \mathrm{m}$, apex cells rhomboid to linear up to $45 \times 3.9$ $\mu \mathrm{m}$. Branch leaves same as stem leaves but slightly smaller in size. Perichaetial bract linear lanceolate to ovate laceolate, long acuminate, nerve less. Seta reddish, up to $8 \mathrm{~mm}$ long, capsule brown, peristome teeth normal and more or less $300 \mu \mathrm{m}$ long. Spores 9.5 $\times 12 \mu \mathrm{m}$, smooth.

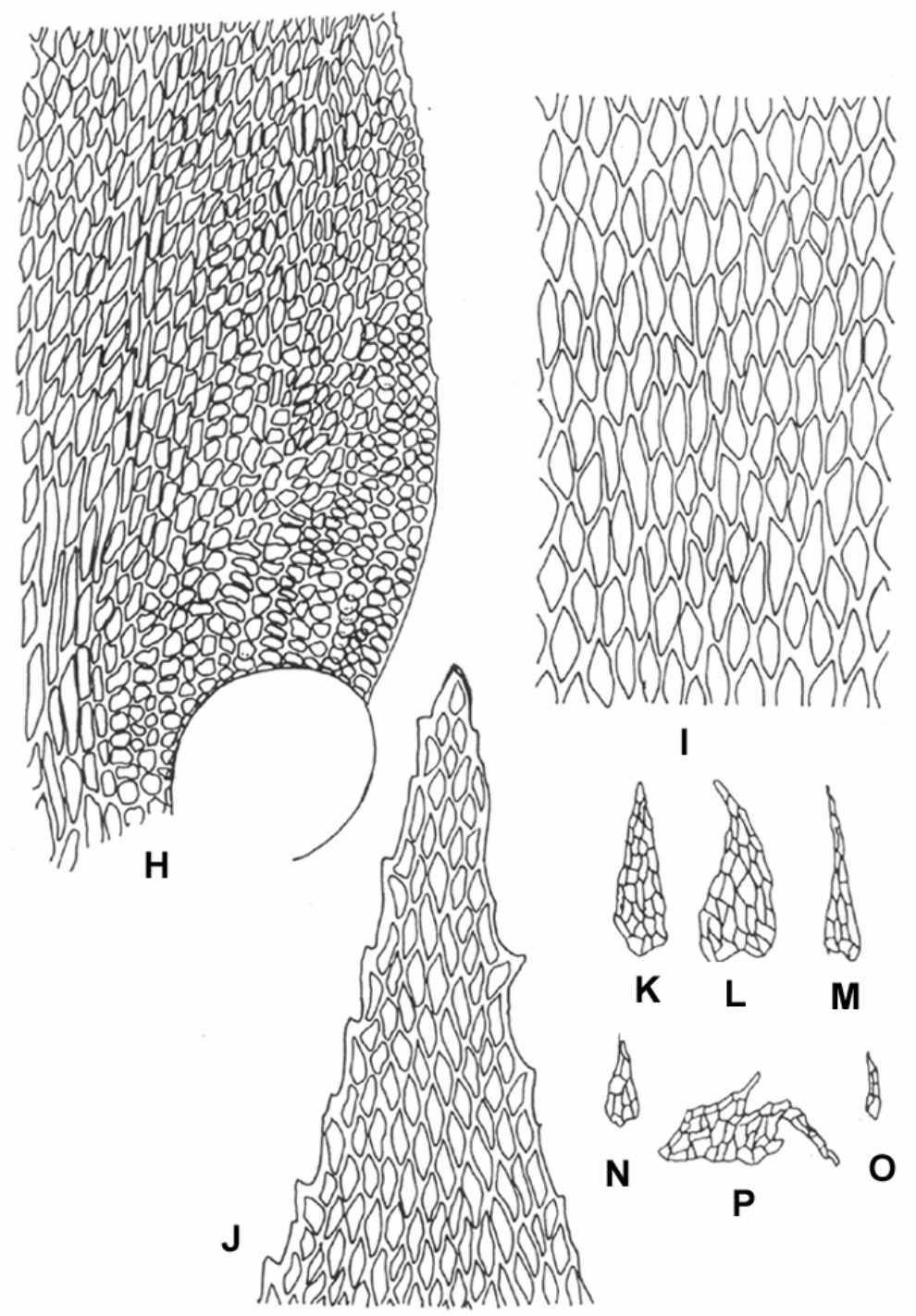

Fig. 3. (Contd.) H. basal laminal cells $(\times 133)$, I. middle laminal cells $(\times 180)$, J. apical laminal cells $(\times 133)$, K-P. paraphyllia $(\times 60)$. 


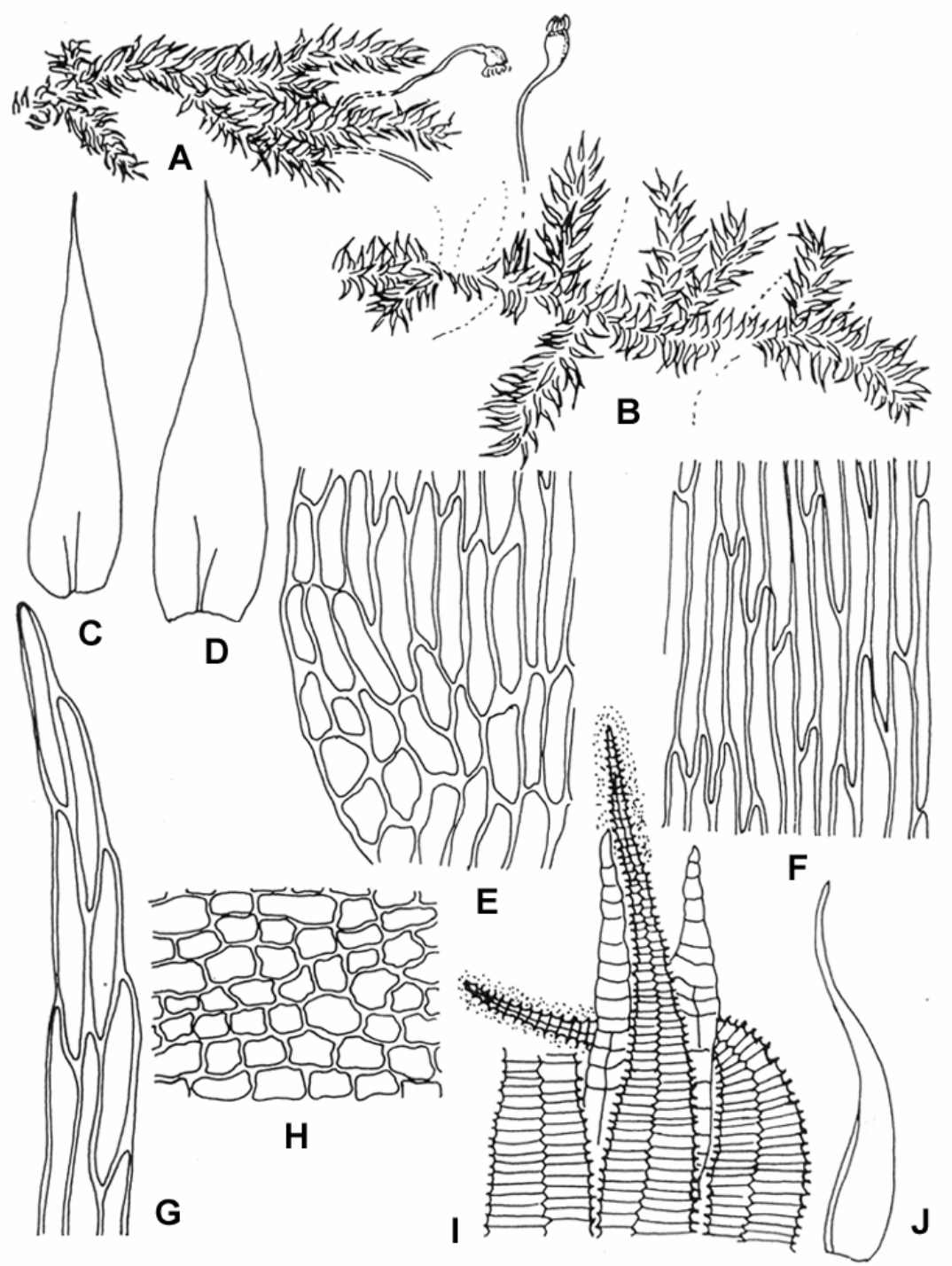

Fig. 4. Campylium gollanii C. Muell. A. dry plant $(\times 6.67)$, B. wet plant $(\times 6.67)$ C, D. leaves $(\times 24)$, E. basal laminal cells $(\times 300)$, F. middle laminal cells $(\times 300)$, G. leaf apex cells $(\times 300)$, H. exothecial cells of the capsule $(\times 80)$, I. peristome teeth $(\times 80)$, J. perichaetial leaf $(\times 24)$.

Specimens examined: Chittagong: Chittagong University Area, on soil, Hamida Khatun, 22.08.93, 279; Cox’s Bazar: Ramu, on soil, M.A. Rahim, 01.03.89, 429; Maulvi Bazar: Srimangal, Madhobkundu, on stone, Hamida Khatun, Abdul Karim, Belal, Syeda Humaira Afroz, Md. Shahabuddin, 28.2.92, 292; 289; Mymensingh: Gouripur, on soil, Jashim Sheikh, 08.02.93, 295; Pabna: Sathia, on soil, Luna Ahmed, 28.08.92, 280; Bera, on soil, Luna Ahmed, 29.08.92; Rangamati: Kaptai, on sandi moist tilla, Hamida Khatun, 20.02.92, 96. 
5. Campylium sommerfeltii (Myr.) Bryhn. Explor. 61. 1893; Kanda in journ. Sc. Hiroshima University Ser. B. Div. 2 (Bot.) 15(2) :255. 1975

(Fig. 5)

Hypnum sommerfeltii Myr. K. Svenska vetensk Akad. Aorsb. 328.1831.

Monoecious, plants in dense carpet, pale green to bright golden green, sometimes brownish in colour. Main stem creeping, more or less $2 \mathrm{~cm}$ long, erect, irregularly branched, 1 to $2 \mathrm{~cm}$ long, densely foliate, branches more or less 4 to $5 \mathrm{~mm}$ long. Leaves

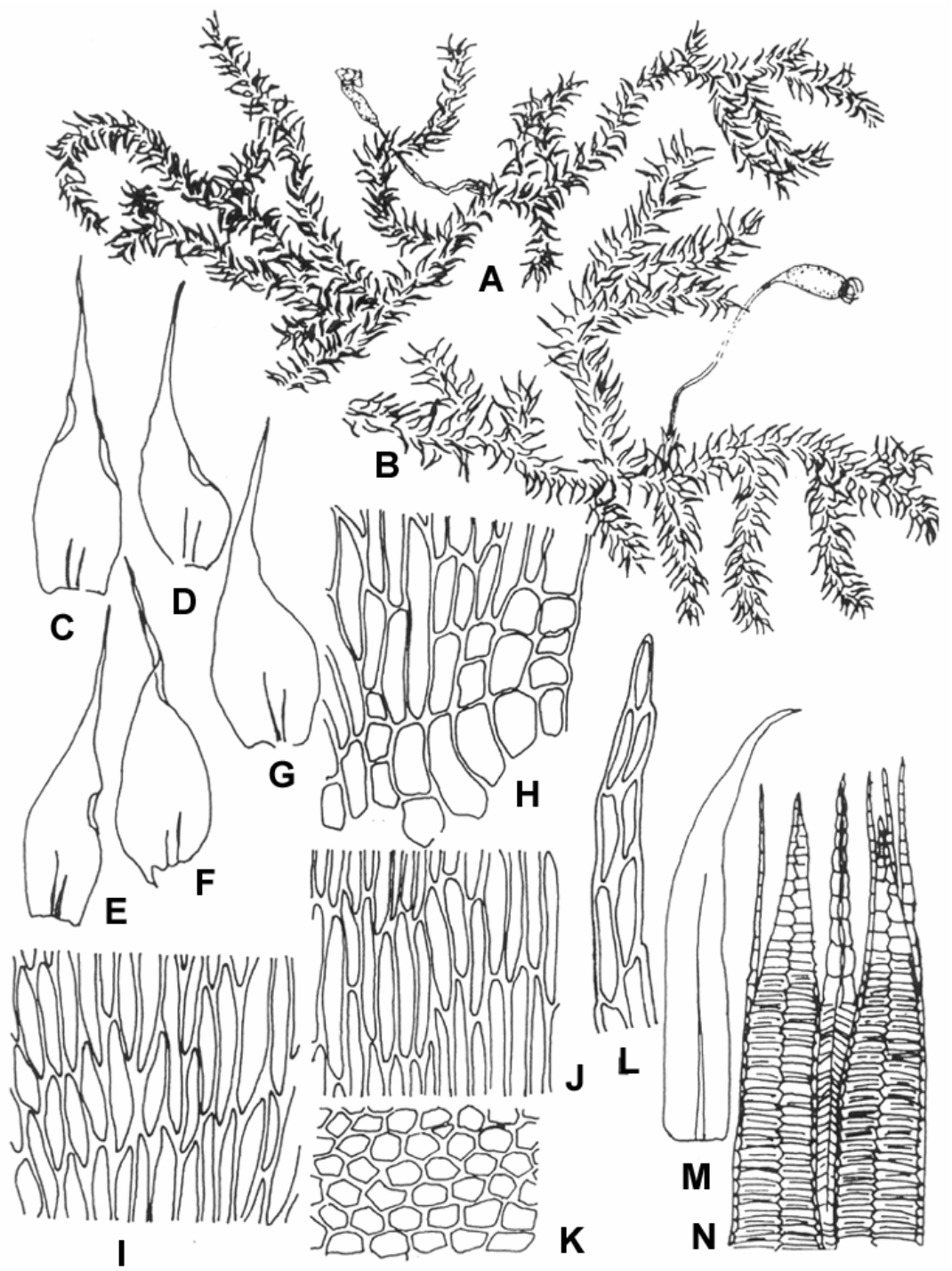

Fig. 5. Campylium sommerfeltii (Myr.) Bryhn. A. dry plant (×6.67), B. wet plant (×6.67), C-G. leaves $(\times 24), \mathrm{H}$. basal laminal cells at the one side of midrib $(\times 300)$, I. basal middle laminal cells at the other side of midrib $(\times 300)$ J. middle laminal cells $(\times 200)$, K. exothecial cells $(\times 133)$, L. apical laminal cells $(\times 200)$, M. perichaetial leaf $(\times 24)$, N. peristome teeth $(\times 133)$. 
more or less 0.91 to $0.95 \mathrm{~mm}$ long, 0.3 to $0.4 \mathrm{~mm}$ wide, concave, ovate, gradually narrowed into a long acumen, margin plain, basal portion slighty dentate, very faintly toothed, entire above, nerve short, double, unequal, reaching up to $2 / 3$ or $1 / 4$ th of leave length. Cells in middle of leaf is variable in size and shape c.77.59 $\times 6.6 \mu \mathrm{m}$, tip cells linear, c. $36.99 \times 3 \mu \mathrm{m}$, angular cells few, alar cells quadrate to sub-rectangular c.36 $\times$ $4.4 \mu \mathrm{m}$. Sporophyte on main stem, perichaetial leaf erect, elongated, c. $1.5 \mathrm{~mm}$ long, nerve present up to $2 / 3$ of its length. Seta brown, erect, c. $1 \mathrm{~cm}$ long, capsule brown , oblong-elongate, exothecial cells irregular in shape, c. 20-30 $\mu \mathrm{m}$ wide, brown and thinwalled. Peristome normal, hypnoid, exostome brown, endstome pale-yellow colour; cilia also present .

Specimen examined: Habiganj: Satchhari, Chunarughat, on the bark of tree, Shafaet Ahmed Khan, 17.10.88, 140.

6. Campylium stellatum (Hedw.) C. Jens. In Lange, Medd. Grönl. 3: 328 , 1887.

Hypnum stellatum Hedw., Sp. Musc. , P. 280, 1801

(Fig. 6)

Campyliodelphus stellatus (Hedw.) Kanda, Jour. Sci. Hiroshima Univ. B2, 15: 269, (1975) 1976.

Plant slender, moderately robust, in loose mats, dioecious, green or yellowish to golden green, more or less glossy when dry. Main stem creeping, irregularly branched, ascending. Leaves crowded, erect to erect spreading from a broad suberect base,c. striolate when dry, 1-2 mm long, 0.3-0.5 mm broad, ovate lanceolate gradually narrowed to a long slender, channeled acumem, acuminating straight, sometimes recurved, margin entire, costa short, double, unequal, sometimes absent, in few reaching about $1 / 3$ or $1 / 4$ th of the leaf length, cells fusiform, smooth, upper cells linear, c. 51 x $4.5 \mu \mathrm{m}$ at tip, c.7.2 $\times$ $3 \mu \mathrm{m}$ at middle, basal cells thick-walled c. $47.7 \times 6.6 \mu \mathrm{m}$ alar cells conspicuously differentiated, large, short, oblong becoming thick-walled c. $14.7 \times 11.76 \mu \mathrm{m}$ and brownish with age. Sporophyte on main stem, perichaetial leaf elongate, errect, nerve less, seta erect, 15-20 mm long, capsule oblong-elongate, 2-3 mm long, c. $1 \mathrm{~mm}$ wide. Peristome normal, exostome yellow brown, endostome pale yellow, cilia also present, basal membrane low.

Specimens examined: Maulvi Bazar: Srimangal, on soil, Hamida khatun, Syeda Humaira Afroz, Md Shahabuddin, Md. Shamim. 29.02.92, 272; Pabna: Atghoria, on soil, Luna Ahmed, 04.03.94, 285. 


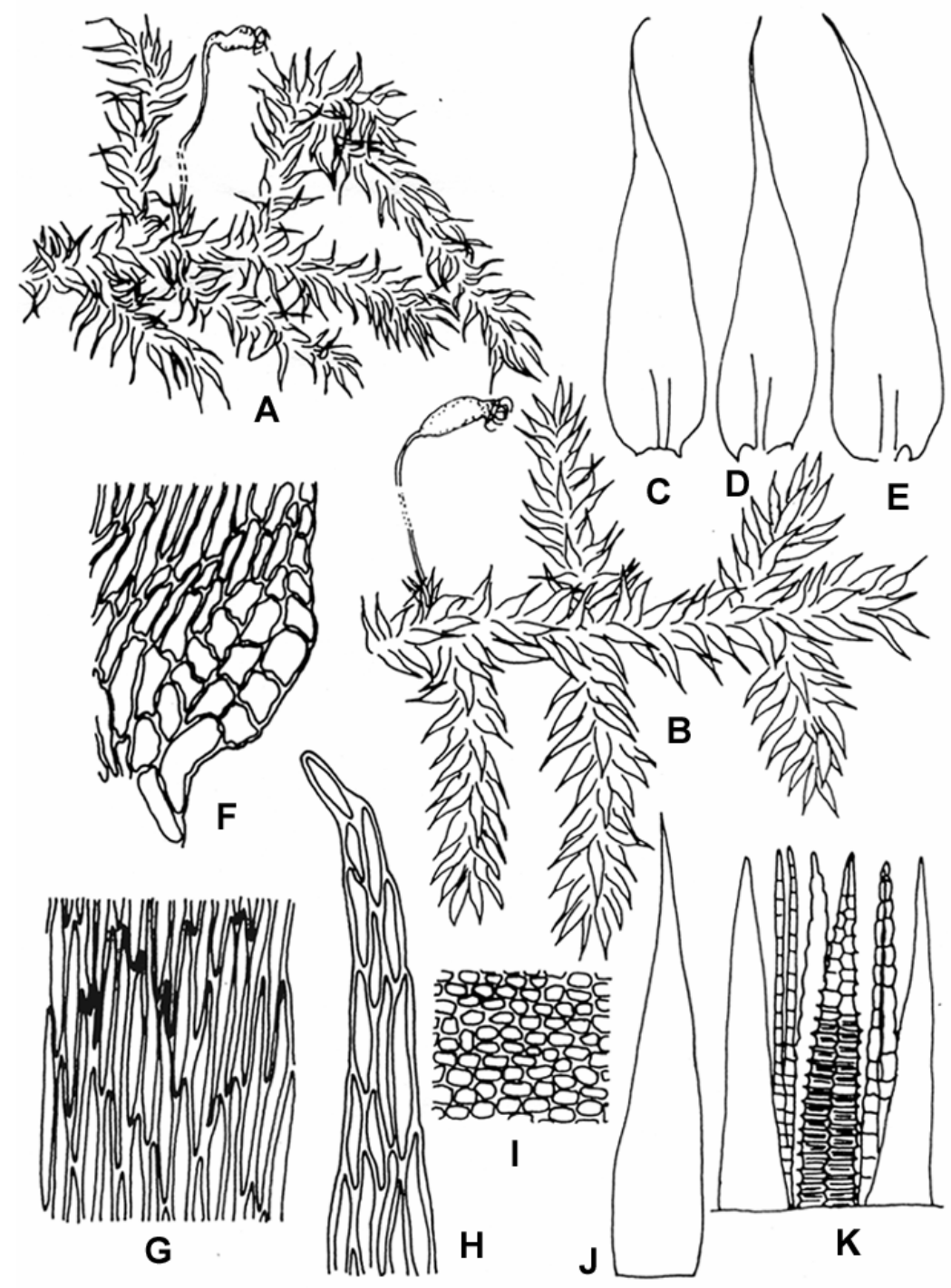

Fig. 6. Campylium stellatum (Hedw.) C. Jens. A. dry plant $(\times 6.67)$, B. wet plant $(\times 6.67)$, C, E. leaves $(\times 24)$, F. basal laminal cells $(\times 300)$, G. middle laminal cells $(\times 33)$, H. apical laminal cells $(\times 300)$, I. exothecial cells of the capsule $(\times 200)$, J. perichaetial leaf $(\times 24)$, K. peristome teeth $(\times 133)$.

\section{Acknowledgement}

The authors are grateful to National Professor A.K.M. Nurul Islam, Department of Botany, University of Dhaka for his valuable suggestions and constructive criticism for the preparation of the manuscript. 


\section{Refereces}

Banu-Fattah, K. 2005. Distichophyllum schmidtii Broth. (Hookeriaceae) - A new report from Bangladesh. Bangladesh J. Bot. 34(1): 45-47.

Chopra, R. S. 1975. Taxonomy of Indian mosses. New Delhi- India. 440-531.

Gangulee, H. C. 1977.Mosses of Eastern India and adjacent regions. A monograph. Fasc. 6. Calcutta , India. 1516-1527.

Gangulee, H. C. 1978.Mosses of Eastern India and adjacent regions. A monograph. Fasc. 7. Calcutta, India. 1657-1661, 1680-1683.

Khatun, H and Hadiuzzaman, S. 1994. Taxonomic studies of some pleurocarpic mosses of Bangladesh. Bangladesh J. Bot. 23(1): 113-122.

Khatun, H and Hadiuzzaman, S. 1995. Addition to the pleurocarpous mosses of Bangladesh. Bangladesh J. Bot. 24(2): 183-191.

Khatun, H and Hadiuzzaman, S. 2003. Pleurocarpous mosses of Bangladesh. Family Neckeraceae-1. Bangladesh J. Plant Taxon. 10(2): 47-55.

Khatun, H and Hadiuzzaman, S. 2004. Pleurocarpous mosses of Bangladesh. Family Neckeraceae-2. Bangladesh J. Plant Taxon. 11(1): 43-47.

Khatun, H and Hadiuzzaman, S. 2004. Pleurocarpous mosses of Bangladesh. Family Erpodiaceae. Bangladesh J. Plant Taxon. 11(2): 29-32.

Khatun, H and Hadiuzzaman, S. 2005. Pleurocarpous mosses of Bangladesh. Family Meteoriaceae and Pterobryaceae. Bangladesh J. Plant Taxon. 12(1): 53-57.

Khatun, H and Hadiuzzaman, S. 2005. Pleurocarpous mosses of Bangladesh. Family Thuidiaceae and Brachytheciaceae. Bangladesh J. Plant Taxon. 12(2): 71-84

Sinclair, J. 1955. Flora of Cox's Bazar, East Pakistan. Bull. Bot. Soc. Bengal, 9(2): 110-111.

Tixier, P. 1967. Bryophytae Indosinicae. Dacca Univ. Stud. Pt. B. 15(1): 1-14. 